\section{P142 HOW CAN PATIENTS SHAPE IBD RESEARCH? FEEDBACK FROM A NATIONWIDE PATIENT INVOLVEMENT EXERCISE}

${ }^{1}$ Kapil Sahnan, Sue Blackwell, Azmina Verjee, ${ }^{2}$ Helen Terry, ${ }^{2}$ Anita Liu, ${ }^{2}$ Nikul Bakshi, ${ }^{2}$ Wendy Childs, ${ }^{3}$ Ramesh Arasaradnam, ${ }^{4}$ Matthew Brookes, ${ }^{5}$ Nicola Fearnhead, ${ }^{6}$ Christine Norton, ${ }^{1}$ Phil Tozer, ${ }^{1}$ Ailsa Hart, ${ }^{7}$ Mark A Samaan*. ${ }^{1}$ St Mark's Hospital, London, UK; ${ }^{2}$ Crohn's and Colitis UK; ${ }^{3}$ University Hospitals Coventry and Warwickshire NHS Trust, Coventry, UK; ${ }^{4}$ The Royal Wolverhampton NHS Trust, Wolverhampton, UK; ${ }^{5}$ Addenbrooke's Hospital, Cambridge, UK; ${ }^{6}$ King's College London, London, UK; ' Guy's and St Thomas', London, UK

\subsection{6/gutjnl-2020-bsgcampus.217}

Introduction Patient involvement in research is essential, not only to give patients a voice regarding how research should be carried out, but also to ensure that studies are investigating clinical outcomes that matter to them and that trial interventions are acceptable.

Methods Our multidisciplinary organising committee included IBD-focussed gastroenterologists and surgeons as well as two IBD patients and members of the research team at the UK's IBD patient charity (Crohn's \& Colitis UK, CCUK). Supported by grants from the BSG, BDRF, NIHR and CCUK, we ran two IBD Patient Research Days (IBDPRDs) to involve large, nationwide cohorts of patients in a broad range of research areas. The first took place in London in May 2018 and the second in Manchester in September 2019.

Patient engagement, information and registration was carried out through CCUK's nationwide network of members. Between the two events, 20 research groups were invited to present their studies to the audience and include specific areas for which they required patient involvement.

Sessions were facilitated by members of the organising committee and digital capacity included online steaming of sessions, digital voting on research questions and feedback.

Results Approximately 80 IBD patients registered for each of the IBDPRDs and purposive sampling helped to ensure a range of demographics and IBD phenotype. Presented studies included national and international trials at various phases, such as SPARE, STOP-COLITIS, ASTIClite, IBD BioResource and IBD-BOOST. Researchers asked for patient involvement on:

- Relevance of research topics and shaping of research questions

- Feedback on study design

- Comments on ethical aspects

- Issues regarding recruitment and retention

- Identification of future research priorities

In addition to participation of patients at the events, we also engaged patients virtually, with the live streamed IBD BioResource session being watched almost 500 times and almost 900 responses being received for the accompanying patient involvement questions.

IBDPRD2019 feedback demonstrated an overall patient rating of 8.7/10 and researcher rating of 9.4/10.

Conclusion Patient involvement can have a powerful and positive influence on the design, conduct and delivery of IBD research. It should ideally include a large number of patients, who represent a broad range of lived IBD experiences. We plan an IBDPRD2020 and would encourage other groups to carry out similar events.

\section{P143 VALIDATION OF THE TWO-ITEM UC PRO2 AGAINST SCCAI, BIOCHEMICAL DISEASE ACTIVITY AND QUALITY OF LIFE}

Mark Samaan*, Georgina Cunningham, Gokul Tamilarasan, Shuvra Ray, Joel Mawdsley, Simon Anderson, Jeremy Sanderson, Peter Irving. Guy's and St Thomas' Hospital, London, UK

10.1136/gutjnl-2020-bsgcampus.218

Background Patient-reported outcomes (PRO) have an increasingly important role in IBD. This is true of monitoring of disease activity in clinical practice and as well as the evaluation of new therapies, when used as clinical trial endpoints. An interim two-item PRO (PRO2) has been developed for UC that ranges from 0-6 and consists of the patient derived items from the Mayo score (rectal bleeding and stool frequency). PRO2 was internally validated against endoscopic outcomes. We aimed to add external validation across a range of clinical, biochemical and quality of life outcomes.

Methods Data was collected as part of the GO-LEVEL study, an open-label, phase IV, investigator-initiated study (NCT03124121) which included a prospective cohort of UC patients commencing golimumab induction therapy, as well as a cross-sectional cohort receiving maintenance treatment. Patients commencing induction therapy all had disease activity objectively confirmed and were evaluated at weeks 6, 10 and 14. Patients receiving maintenance therapy were recruited either at the point of flare, or during stable remission. Clinical disease activity was evaluated using PRO2 and SCCAI, quality of life using IBD-Control as well as a $100 \mathrm{~mm}$ visual analogue scale (IBD-Control-VAS), and biochemical activity using FC and CRP.

Clinical remission was defined as a score of $<3$ using SCCAI, or 0 using PRO2.

Correlations were calculated using Spearman's correlation coefficient $\left(r_{s}\right)$ and contingency table analyses using Chi square.

Results GO-LEVEL included a total of 112 patients across the two study cohorts. A total of 217 PRO2 assessments were made with concurrent SCCAI and IBD-Control scores available at all timepoints. CRP measurements were available at 214 of these and FC at 207. Strong correlations were observed between PRO2 and SCCAI $\left(\mathrm{r}_{\mathrm{s}}=0.94, \mathrm{p}<0.0001\right)$, as well as with IBD-Control $\left(\mathrm{r}_{\mathrm{s}}=-0.82, \mathrm{p}<0.0001\right)$ and IBD-Control-VAS $\left(r_{s}=-0.78, p<0.0001\right)$. Significant correlations were also seen between PRO2 and FC $\left(\mathrm{r}_{\mathrm{s}}=0.38, \mathrm{p}<0.0001\right)$ as well as PRO2 and CRP $\left(r_{\mathrm{s}}=0.31, \mathrm{p}<0.0001\right)$.
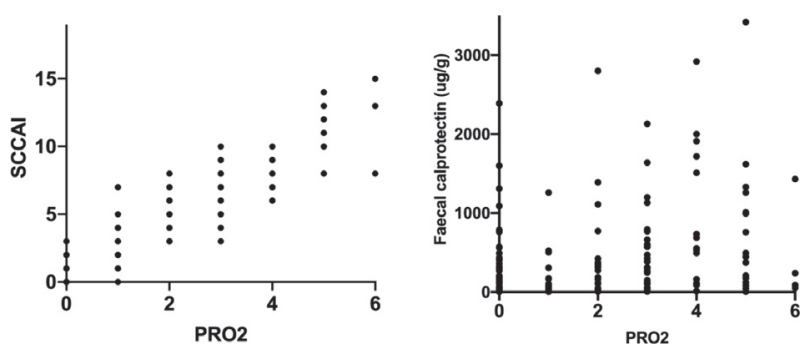

Abstract P143 Figure 1 Scatter plots of PRO2 against SCCAI (left panel, $r_{s}=0.94, p<0.0001$ ) and faecal calprotectin (right panel, $r_{s}=0.38$, $\mathrm{p}<0.0001)$ 